\title{
Unusual chromatin structural organization in the sperm head of a murid rodent from Southern Africa: the red veld rat, Aethomys chrysophilus type B
}

\author{
W. G. Breed \\ Department of Anatomical Sciences, University of Adelaide, South Australia 5005, Australia
}

\begin{abstract}
The structural organization of the chromatin of cauda epididymal spermatozoa of the red veld rat Aethomys chrysophilus type B was investigated by fluorescence microscopy after staining with DNA specific dyes and by transmission electron microscopy after incubation with Triton $X_{100}$, dithiothreitol, and SDS. Staining with DNA dyes showed variation in intensity of fluorescence of the sperm chromatin, with an anterior spherical region staining far more intensely than the surrounding chromatin. Transmission electron microscopy of these spermatozoa indicated that this region was composed of cords and fibres. This chromatin region dispersed more readily than the surrounding chromatin when spermatozoa were incubated with the detergents, and it is suggested that, unlike the rest of the sperm chromatin, it may be a histone-rich region, with protamine(s) being either scarce or absent.
\end{abstract}

\section{Introduction}

In eutherian mammals, there is a marked reduction in nuclear volume during development of the sperm head from a round spermatid, which is formed after the completion of the two meiotic divisions, to a bilaterally flattened, spatulate or falciform structure (Bedford and Hoskins, 1990; Eddy and O'Brien, 1994; Yanagimachi, 1994). During this development, histones are generally removed and replaced by transitional proteins, which are in turn replaced by arginine-rich, basic protamines of low molecular mass. Coincident with this change in nuclear proteins and reduction in nuclear volume, the sperm chromatin becomes highly condensed and homogeneous in appearance when observed with the transmission electron microscope (TEM); thus, its substructural organization becomes unresolvable in conventional TEM thin sections. Only after treatment with detergents and disulfide reducing agents can some substructure of the chromatin of the sperm nucleus be observed (Calvin and Bedford, 1971; Bedford et al., 1973a, b; Bedford and Calvin, 1974; Zirkin et al., 1985 for review).

In a few species of mammal (for example, humans and gorillas (Bedford et al., 1973a; Seuanez et al., 1977) and a few species of murid rodents), highly pleiomorphic populations of spermatozoa are present within the cauda epididymides and vacuoles of various sizes may be found in some sperm nuclei. Among murid rodents, nuclear vacuoles were first reported in the cauda epididymal spermatozoa of the Australian heath rat Pseudomys shortridgei (Breed, 1983) and have been documented for the spermatozoa of two species of Southern Asian bandicoot rats, Bandicota savilei and B. indica (Breed, 1993). A fourth species of murid rodent, (recently reclassified as Aethomys ineptus, Aethomys chrysophilus type B. Chimimba, 1977) from Southern Africa, has been found to have cauda

Received 24 April 1997. epididymal spermatozoa with very different morphology from that of most murid rodents, with evidence of pleiomorphism (Gordon and Watson, 1986; Visser and Robinson, 1987; Breed et al., 1988). These spermatozoa have very large and complex acrosomes and do not appear to have uniform, electron dense, nuclei (Breed ef al., 1988; Breed, 1995). The purpose of the present study was to investigate the structural organization of the chromatin in these sperm nuclei by staining with DNAspecific dyes and by examining the effects of incubation in either detergents or the disulfide reducing agent, dithiothreitol.

\section{Materials and Methods}

Two groups of mature male red veld rats, Aethomys chrysophilus type B, were used. The first group consisted of five individuals collected at various localities in Vaalwater, South Africa in January 1995 and kept at the Transvaal Museum in Pretoria. Two additional adult males were caught in the wild at this time and killed within 2 days of capture; one came from Tswaing Crater, N.W. Province and the other from near Vaalwater, Waterberge, Northern Province. The second group of five animals was collected at Tswaing Crater in late August 1996 and these animals were killed within 4 days of capture.

Examination of sperm motility indicated that three of the captive kept animals in the first group and one wild-caught individual in the second group had immotile spermatozoa; therefore, these four animals were excluded from the study. The following experiments were performed on the cauda epididymal spermatozoa from the other individuals.

Spermatozoa were extruded from the caudae epididymides into $0.1 \mathrm{~mol}$ phosphate buffer $\mathrm{I}^{-1}$ and pelleted. The supernatent was aspirated off and the spermatozoa were incubated at room temperature in (1) $0.5 \%$ or $0.1 \%(\mathrm{w} / \mathrm{v}) \mathrm{SDS}$ in $0.1 \mathrm{~mol}$ phosphate buffer $\mathrm{I}^{-1}, \mathrm{pH} 7.4$ for $30 \mathrm{~min}$; (2) $0.5 \%(\mathrm{w} / \mathrm{v})$ 
dithiothreitol (DTT) made up in borate buffer, $\mathrm{pH} 9$; or (3) $0.1 \%$ or $0.5 \%(\mathrm{v} / \mathrm{v})$ Triton X100 in $0.1 \mathrm{~mol}$ phosphate buffer $l^{-1}$ for 30 or $60 \mathrm{~min}$.

At the completion of all incubations, samples of spermatozoa were centrifuged at about $1700 \mathrm{~g}$ for $4-10 \mathrm{~min}$, the supernatant removed, and $3 \%$ glutaraldehyde $/ 3 \%$ paraformaldehyde in 0.1 mol phosphate buffer $1^{-1}, \mathrm{pH} 7.4$, was added. The fixed pellets were returned to Adelaide by air, where the material was prepared for TEM. For this, the samples were post-fixed for 5-15 min with $1 \%(\mathrm{v} v)$ osmium tetroxide, rinsed in buffer, dehydrated, and then embedded in Araldite. Subsequently, $0.5-1.0 \mu \mathrm{m}$ and ultrathin sections were cut. The ultrathin sections were stained with uranyl acetate and lead citrate and examined with a Philips CM 100 TEM.

In addition to the above experiments, small pieces of cauda, proximal and distal vasa deferentia were immersed in TEM fixative at the time the animals were killed. After returning the tissue to Adelaide, some samples were prepared for TEM as above. From a few of the cauda and vas deferens samples, spermatozoa were extruded onto a few drops of fixative placed on microscope slides. Various concentrations of the DNA fluorochromes, SYBR Green I or SYBR Green II (Molecular Probes, Eugene, OR), were added to these spermatozoa, or the cauda spermatozoa were stained with about a 1:1000 dilution of the dimethyl sulfoxide (DMSO) stock solution of Pico Green (Molecular Probes). These spermatozoa were examined with a phase-contrast and fluorescent light microscope using fluorescein excitation $(488 \mathrm{~nm})$ and an emission wavelength of $525 \mathrm{~nm}$.

\section{Results}

\section{Light microscopy}

Phase-contrast microscopy indicated similar head morphology of spermatozoa obtained from the cauda epididymides and all regions of the vas deferens. The sperm head, although somewhat variable in shape, was bilaterally flattened, disc or wedge-shaped and tapered a little towards the connecting piece. The apical tip of the sperm head was often seen to be reflected caudally (Fig. $1 \mathrm{a}-\mathrm{c}$ ). The posterior third of the sperm head was sometimes more refractile than the anterior region, although within the anterior region a highly birefrigent, spherical, focal area normally occurred that varied in position among spermatozoa (Fig. Ia-c).

Fluorescent microscopy, after staining with both the SYBR Green I and II and with the Pico Green DNA fluorochromes, showed that both the basal third of the sperm head and the birefrigent focal region in the anterior part of the sperm head fluoresced much brighter than the rest of the nucleus (Fig. 2a-c), with the position of the bright fluorescent spot varying from central to eccentric.

\section{Transmission electron microscopy of spermatozoa from the cauda epididymides and vasa deferentia}

Low power transmission electron microscopy indicated that, in sections cut through the cauda epididymides and vasa deferentia, the sperm heads varied considerably in their structural organization, with the nucleus showing variable degrees of chromatin condensation. A few large round cells,
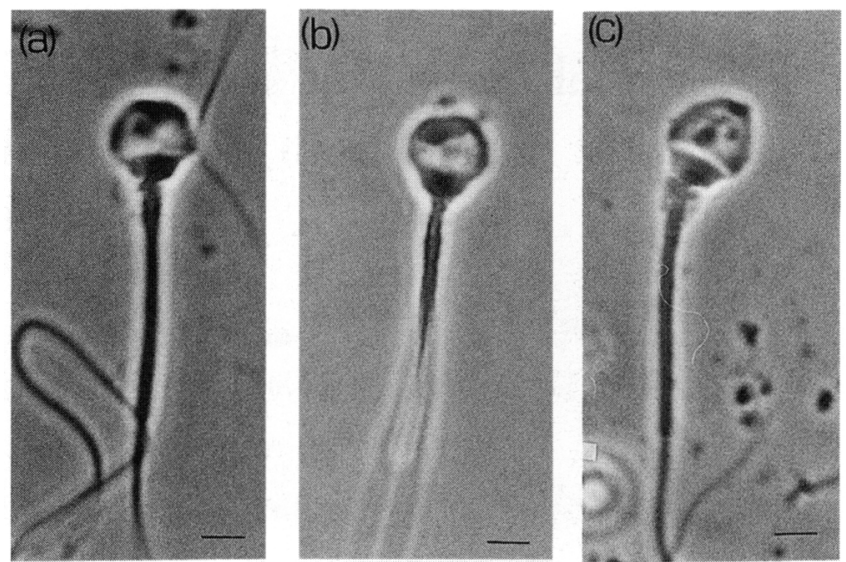

Fig. 1. Phase contrast micrographs of typical cauda epididymal spermatozoa from Aethomys chrysophilus type B show a highly birefrigent, spherical focal anterior region and a more refractile basal region of the sperm head. The apical tip of the sperm head, which is part of the acrosome, is reflected caudally (see Fig. 3b). Scale bars represent $2.0 \mu \mathrm{m}$.
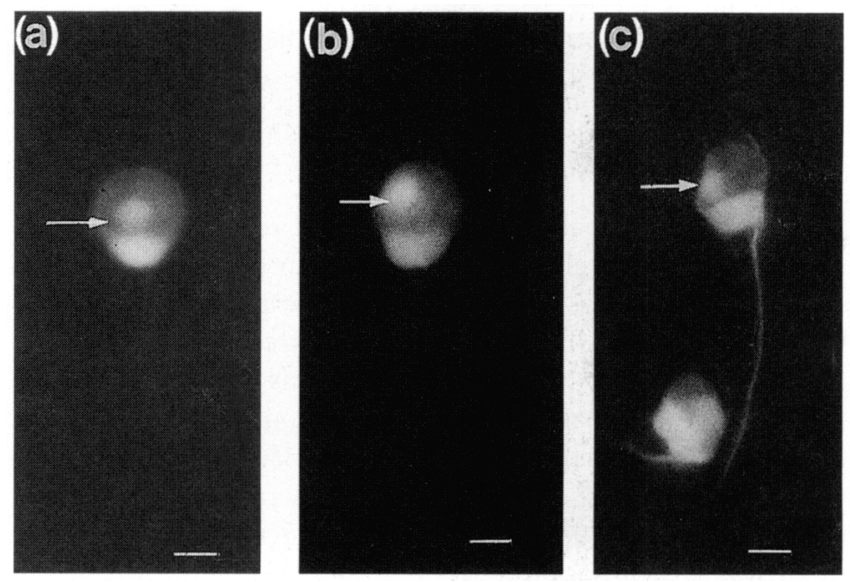

Fig. 2. Fluorescent micrographs of typical cauda epididymal spermatozoa from Aethomys chrysophilus type B after staining with SYBR Green II, showing that the basal part of sperm head and an anterior spherical region (arrows) fluoresce more brightly than the rest of the chromatin. Scale bars represent $2.0 \mu \mathrm{m}$.

presumably spermatocytes, were usually present. By far the majority of germ cells had a bilaterally flattened, wedge-shaped sperm head and a nucleus with localized regions of chromatin where threads or cords could be seen (Fig. 3a-d). The nucleus was capped anteriorly by a large acrosome that had a very large, caudally reflected, apical segment (Fig. 3b). In the anterior part of the nucleus there was generally a peripheral region of chromatin, >100 nm thick, which was uniformly electron dense. Within this region, the chromatin often appeared as interwoven cords between 20-40 nm diameter (Fig. 3b-d), in between which were fibres of $7-10 \mathrm{~nm}$ diameter. Other sperm nuclei had cords and fibres of varying sizes (Fig. 3d). The posterior region of the nucleus was either uniformly electron dense or composed of a mass of $60-120 \mathrm{~nm}$ cords with $5-20 \mathrm{~nm}$ fibres occasionally evident between the cords. 

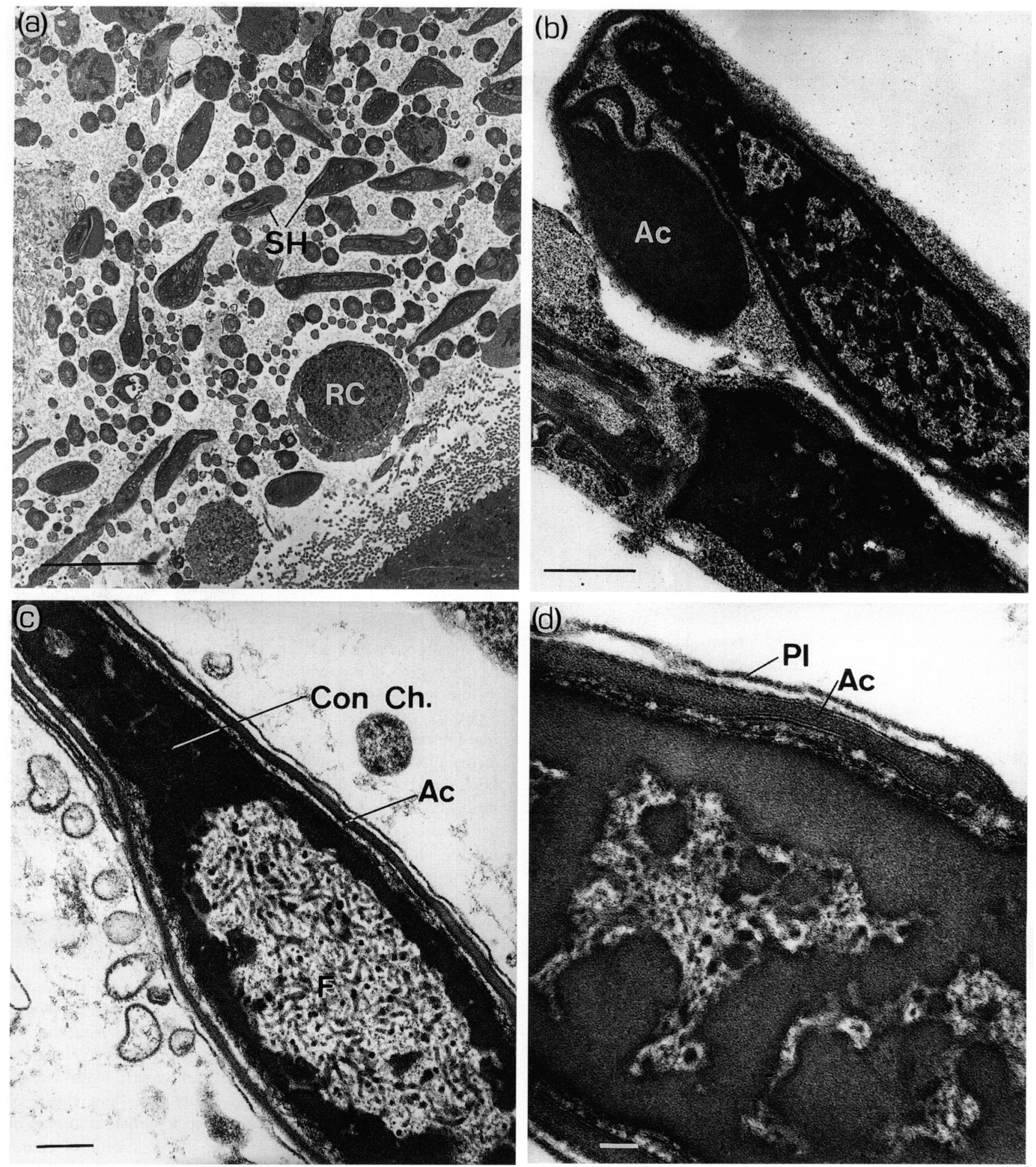

Fig. 3. Transmission electron micrographs of spermatozoa of Aethomus dirusophints type B from (a, c, d) cauda epididymides and (b) vas deferens. In the low power micrograph (a), two large round cells (RC), presumably spermatocytes, can be seen in the lumen together with thin sections through many sperm heads (SH) and tails; most of the sperm heads contain a nucleus that does not have homogeneous electron-dense chromatin Higher power TEM (b) through a sperm head from vas deferens, showing a huge apical segment of acrosome (Ac) and a nucleus in which the chromatin is partly composed of interwoven cords. In many sperm nuclei (c) a central region of $20-30 \mathrm{~nm}$ fibres (F) was present with the peripheral region, composed of homogeneous, clectron dense, material. Anteriorly, typical condensed chromatin (Con Ch.) occurs. In other sperm nuclei (d) larger cords are intermixed with smaller fibres. Pl: plasmalemma. Scale bars represent $5 \mu \mathrm{m}$ (a); $500 \mathrm{~nm}$ (b); $200 \mathrm{~nm}$ (c); and $50 \mathrm{~nm}$ (d). 
Transmission electron microscopy of cauda epididymal spermatozoa after incubation with detergents

Incubation with $0.1 \%$ or $0.5 \%$ Triton $\times 100$ at room temperature for $30 \mathrm{~min}$ had an effect on the structural integrity of the cauda epididymal spermatozoa. Most of the acrosomal contents became dispersed, but the inner acrosomal membrane appeared more resistant (Fig. 4c). Between this and the outer nuclear envelope, some granular material was evident in a modestly developed subacrosomal space. In some spermatozoa, both the chromatin of the electron dense region, as well as of the region where 60-120 nm cords were present, appeared unaffected by the detergent, but other spermatozoa had a large, clearly delineated, empty, intranuclear vacuole that was not apparent in the control unincubated spermatozoa (Fig. 4a, b). The location of this vacuole was in the region that in control spermatozoa often contained visible cords and fibres. Therefore, it appeared that the chromatin of these spermatozoa had become dispersed by the detergent.

Incubation in dithiothreitol also dispersed the acrosomal contents but generally left the chromatin intact (Fig. 4d).

The effect of incubation of spermatozoa in either $0.1 \%$ or $0.5 \%$ SDS gave similar results but these were markedly different from the effects of Triton X100 or DTT. Significant differences in the effect of both concentrations of SDS on the chromatin of individual spermatozoa occurred in all samples. In some, much of the chromatin remained fairly electron dense but occasionally had a rather granular appearance, although a large empty vacuole was often evident (Fig. 5a). In other, often adjacent, spermatozoa, which had been subjected to almost identical conditions, the chromatin had become greatly expanded and decondensed to varying degrees (Fig. 5a, b).

Where chromatin decondensation had taken place, there was often a differential effect between the various regions of the nucleus. In some, the chromatin in the basal part of the sperm nucleus formed thin, $3-20 \mathrm{~nm}$, filaments, whereas in the anterior part, much of the chromatin had dispersed into $30-60 \mathrm{~nm}$ cords and $3-5 \mathrm{~nm}$ fibres, although a peripheral rim of $30-70 \mathrm{~nm}$ thick electron dense chromatin generally remained (Fig. 5c, d). Occasionally, a small region of granular chromatin was evident within a large nuclear vacuole (Fig. $5 \mathrm{~d}$ ).

\section{Discussion}

Transmission electron microscopy of spermatozoa of numerous species of eutherian mammals has demonstrated the almost universal occurrence of a sperm head with a nucleus the chromatin of which is uniformly very electron dense. No substructure is evident, although incubation in detergents and disulfide reducing agents can cause much swelling of the nucleus, with the result that the chromatin appears to be composed of fibres or cords anchored to a fibrous nuclear matrix and an annulus that lies near the connecting piece of the sperm tail (Ward and Coffey, 1989, 1991; Barone et al., 1994). However, humans and gorillas (Bedford et al., 1973a, b; Seuanez et al., 1977) differ from most species in having pleiomorphic spermatozoa, some of which possess conspicuous nuclear vacuoles. In the majority of species of murid rodents, the sperm head is falciform or hook-shaped and the nucleus consists of homogenous, electron-dense material that extends into the apical hook. In addition, sperm populations for any one species are remarkably consistent in form, so that the sperm head shape is characteristic for the particular species (Friend, 1936; Bishop and Austin, 1957; Fawcett, 1970; Bedford and Hoskins, 1990). However, there are a few species of murids in which highly divergent sperm head morphology has evolved, and in which populations of spermatozoa are highly pleiomorphic and, like those of humans and gorillas, often have nuclear vacuoles, for example, Pseudomys shortridgei of southern Australia (Breed, 1983, in press), and Bandicota indica and B. savilei of southern Asia (Breed, 1993).

Spermatozoa of the species investigated in the present study, the red veld rat (Aethomys chrysophilus type B) from southern Africa, appear to be unusual in several respects. Sperm populations are highly variable, regardless of whether the animals have been caught in the wild or bred in the laboratory, and many of the spermatozoa appear pathological, with grossly abnormal acrosomes, enlarged foci of subacrosomal material, two heads attached to the one tail or, conversely, one head attached to two tails. In addition, a few, large round cells with vesicular nuclei, which are presumably primary spermatocytes, are occasionally evident. However, the spermatozoa that appear to be 'normal' in their arrangement of organelles have two unique features that set them apart from the spermatozoa of almost all other mammals, including those of all murid rodents described to date. First, they have an acrosome with a massive apical segment that appears to undergo flexion during epididymal transit (Breed et al., 1988). Second, part of the chromatin of cauda epididymal spermatozoa usually appears to have a region of recognizable fibres and cords when observed in thin sections with the TEM. Similar results have also been found for spermatozoa from the vas deferens, which suggests that the chromatin does not continue to undergo condensation as the spermatozoa travel further along the male reproductive tract. The chromatin in the region where cords and fibres are visible fluoresces much more brightly with DNA dyes than the surrounding chromatin, indicating that a greater concentration of dye molecules occurs in this region after staining. This finding suggests that either there is more DNA in this area (a rather unlikely event), or that the DNA has more binding sites for, or is more accessible to, the DNA dyes, perhaps as a result of a less tight packaging of the chromatin fibres and, hence, more exposed sites for intercalation of the dye molecules.

In eutherian mammals, the tight packaging of the chromatin and the cross-linking of the protamines by disulfide bonds results in cauda epididymal sperm chromatin being highly resistant to dispersion by mild detergents. However, there are considerable species differences in this regard, with human spermatozoa being more easily solubilized than those of laboratory rats and bulls. This interspecific variation in nuclear stability of spermatozoa may relate to the extent of sperm nuclear disulfide bonding and, possibly, to differences in the protamines present (Bedford et al., 1973a, b; Mahi and Yanagimachi, 1975; Calvin, 1976; Perreault et al., 1988). The nuclei of cauda and washed, ejaculated human spermatozoa are also unusual in exhibiting a variable response to SDS, with some decondensing when incubated in SDS alone, and others remaining unchanged (Bedford et al., 1973a, b; Blazak and Overstreet, 1982). This variable response may be due to 

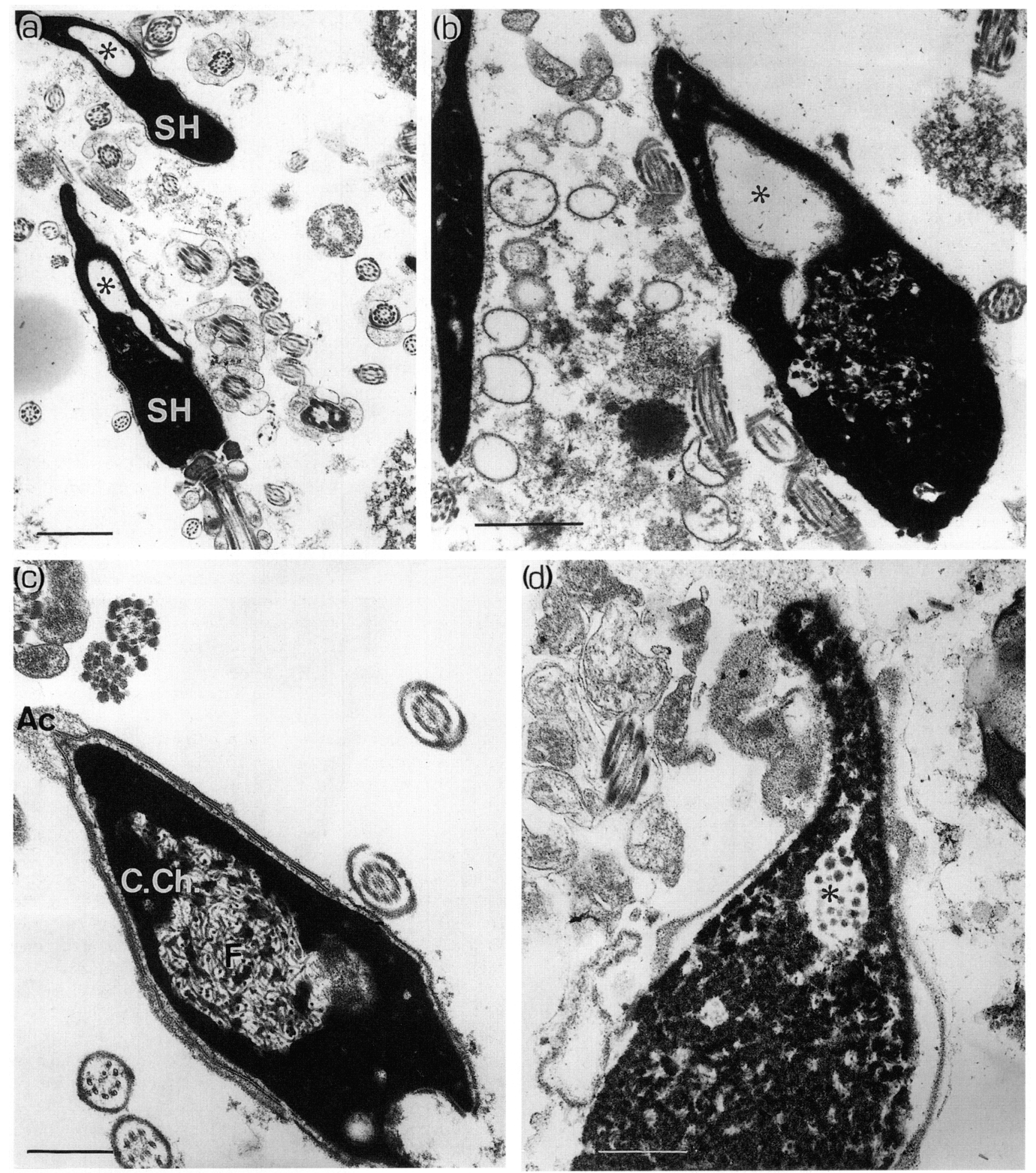

Fig. 4. Transmission electron micrographs of sperm heads $(\mathrm{SH})$ of cauda epididymidal spermatozoa from Aethomys chrysuphilus type B after incubation in $0.1 \%$ Triton $X 100$ for $30 \mathrm{~min}(\mathrm{a}, \mathrm{b}, \mathrm{c})$ or $0.5 \%(\mathrm{w} / \mathrm{v})$ dithiothreitol in borate buffer, $\mathrm{pH} 9$ (d). In all cases, the acrosome has largely dispersed. In the spermatozoa treated with Triton $X_{100}$, some $(a, b)$ have a large central vacuole in the nucleus, which presumably had threads and cords of chromatin before incubation in the detergent, whereas in other spermatozoa (c) these are still present and surrounded by condensed chromatin $(\mathrm{C} . \mathrm{Ch})$. In the spermatozoa incubated with DTT (d), the chromatin is still largely condensed, and a small localized region of cords $\left({ }^{*}\right)$ is evident. Scale bars represent $1 \mu \mathrm{m}(\mathrm{a}, \mathrm{c}) ; 500 \mathrm{~nm}(\mathrm{~b}, \mathrm{~d})$. 

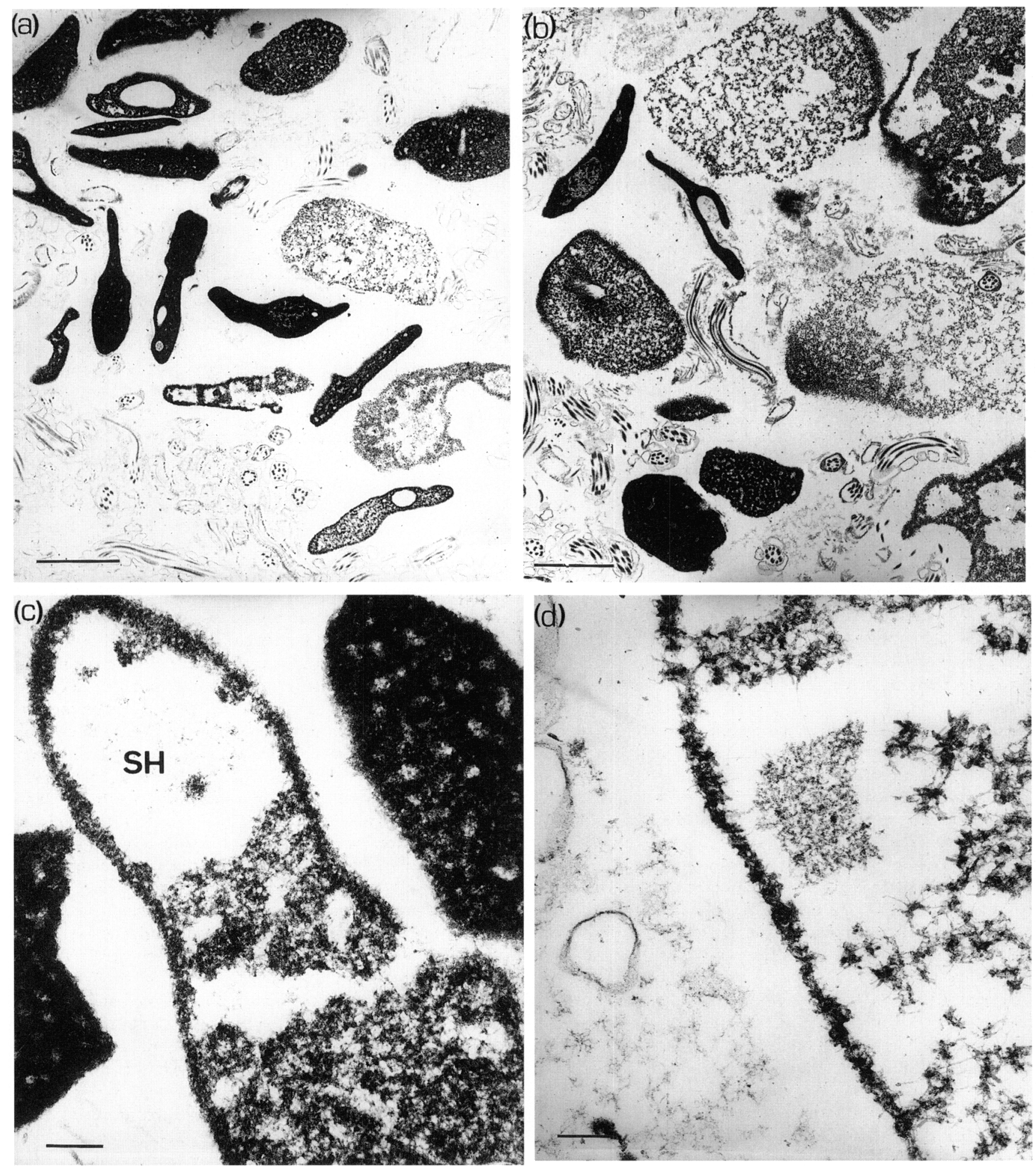

Fig. 5. Transmission electron micrographs of cauda epididymal spermatozoa from Aethomys chrysophilus type B after incubation in $0.1 \%$ (w/v) sodium dodecyl sulfate. Markedly different amounts of chromatin dispersion were invariably found between the individual spermatozoa ( $a$, b), with some having condensed chromatin, albeit with a large nuclear vacuole, whereas other spermatozoa were swollen and possessed decondensed chromatin. In some spermatozoa, the basal part of the nucleus had more dispersed chromatin than the anterior region (b). In the anterior region, there was often a rim of chromatin (c) around dispersed fibres which sometimes contained a granular mass of chromatin within the large nuclear vacuole (c, d). SH, sperm head. Scale bars represent $2 \mu \mathrm{m}$ (a, b); I $\mu \mathrm{m}$ (c); and $200 \mathrm{~nm}$ (d). 
variation in the extent of the oxidation of the sulfydryl groups between the chromatin fibres of the spermatozoa nuclei. The present study demonstrates that, unlike cauda spermatozoa of most eutherians where both SDS and DTT are required to disperse the chromatin (Calvin and Bedford, 1971), SDS alone was sufficient for chromatin dispersion in most veld rat spermatozoa from the cauda, although, as in human spermatozoa, the different individual spermatozoa varied greatly in their response to the SDS. Furthermore, in the spermatozoa whose electron dense chromatin did not decondense when incubated with SDS, the central region of anastomosing chromatin fibres usually dispersed and dispersion was even brought about in some spermatozoa by incubation in $0.1 \%$ Triton X100. This finding suggests that there may be a variable arrangement, or amount, of protamine bonding between the chromatin fibres in different spermatozoa and that the central region, where the cords and fibres are visible in unincubated spermatozoa, may lack disulfide-bonded chromatin.

Although the usual keratinoid nature of the eutherian cauda spermatozoon nucleus is the result of disulfide bonds between and within the protamine molecules (Calvin and Bedford, 1971; Bedford and Calvin, 1974), some histones are also known to be present in small amounts in the nuclei of the spermatozoa of bulls and men. In red veld rats, the central chromatin region, which readily disperses, contains chromatin composed of cords $20-50 \mathrm{~nm}$ in diameter interspersed with thinner fibres of $7-10 \mathrm{~nm}$ in diameter. Chromatin in somatic cells is generally composed of $10 \mathrm{~nm}$ fibres packed into $30 \mathrm{~nm}$ wide threads perhaps in the form of a solenoid (Finch and Klug, 1976). Such packing is very different from that of the nuclei of eutherian spermatozoa, where the tertiary structure of protamine-rich DNA appears to be in the form of toroids or loop doughnuts (Ward, 1993) or lamellae (Koehler et al., 1983). However, the size of the cords and fibres of the central region of the sperm nucleus of red veld rats is similar to that of the fibres in somatic cell nuclei. Therefore, it is possible that the chromatin is histone-rich in this region and that protamines are either scarce or non-existent. The fact that this region of the sperm nucleus also fluoresces more intensely with the DNA dyes than the surrounding region of chromatin suggests that there is greater access of the DNA to the fluorochromes here than elsewhere in the anterior region of the nucleus. This finding supports the view that the chromatin may not be as tightly compacted by protamines in this region of the sperm nucleus as in the surrounding chromatin.

Aethomys chrysophilus type B is a widely distributed species that occurs throughout much of the eastern region of South Africa, where it is present in grassland, scrub, or savanna woodland with thick undercover (DeGraaff, 1981; Skinner and Smithers, 1990). It has an almost identical external morphology to Aethomys chrysophilus type A from which it has only recently been separated (Chimimba, 1977). The unique form of the spermatozoon is totally different in its morphological characteristics from the spermatozoa of the other Aethomys species, including that of $A$. chrysophilus type $\mathrm{A}$, and any other species of mammal that has been described. The spermatozoa of $A$. chrysophilus type B have a morphology in which the shape of the nucleus, acrosome, perforatorium, as well as sperm tail length, all differ markedly from those of the other species in this genus, in which typical falciform sperm heads occur (Visser and Robinson, 1987; Breed et al., 1988; Breed, 1995). The variable nature of the sperm population and the presence of what are clearly pathological germ cells in the epididymides of these animals suggest that the factors that control sperm chromatin packaging, condensation, maturation and release are not as tightly regulated in this species as is generally the case. Why spermatozoa with these morphological characteristics have evolved in this species remains an enigma, but their existence demonstrates that evolution of a sperm type that exhibits marked pleiomorphism, including many pathological types, and in which populations of cauda epididymal spermatozoa do not have homogeneous electron dense chromatin, can take place in a naturally occurring species of eutherian mammal. To date, no studies appear to have been carried out on the genetic heterozygosity of these animals, but at the present time there is no suggestion that the animals are unusually inbred or that the males are in any way subfertile, as is the case in a few species of large cats where highly pleiomorphic sperm populations occur (Wildt et al., 1983, 1988; Wildt, 1994).

These present findings, together with those on the spermatozoa of two species of Asian bandicoot rats (Breed, 1993), suggest that within the family of murid rodents sperm characteristics can evolve that include marked divergence in form from the usual type of spermatozoon, and in which marked pleiomorphism and a high incidence of abnormal spermatozoa are present in sexually mature males. Spermatozoa from laboratory rats have developed successfully within the testis of an immunodeficient laboratory mouse with a sperm morphology typical of the donor species, indicating that the Sertoli cells are permissive in their support of developing germ cells the shape of which is determined by expression of developing germ cell genes (Clouthier et al., 1995). Furthermore, by expression of an avian protamine gene in male germ cells of laboratory mice, it has been found that, although abnormal sperm chromatin condensation occurred, some of these spermatozoa were able to fertilize ovulated oocytes with resultant normal embryogenesis (Rhim et al., 1995). In addition, in laboratory mice, normal young individuals develop after intracytoplasmic injection of grossly abnormal spermatozoa (Burruel et al., 1996). In conclusion, it appears that, in the Aethomys lineage, a mutation has occurred in the gene(s) that controls the final spermatozoon form and which is expressed within the developing germ cells, with the result that a different sperm morphotype develops in $A$. chrysophilus type B than does in its congeners. This highly derived and variable sperm type, which is a characteristic feature of this species, may not exhibit the normal tight packing of the chromatin by protamines throughout the nucleus. Studies on the genetic control of morphogenesis of the spermatozoon head, the selection of spermatozoa within the female reproductive tract and on the processes of sperm-egg interactions in these animals need to be performed to shed further light on the significance of this highly unusual type of spermatozoon that has evolved in this otherwise typical murid rodent of southern Africa.

The author thanks $\mathrm{C}$. Leigh for carrying out the cutting of sections for transmission electron microscopy, C. Chimimba and G. Bronner of the Transvaal Museum, Pretoria, South Africa for assistance in 
collecting material, A. Breed for assistance with incubations, and E. Breed for typing the manuscript. This study was carried out while the author was on study leave from the University of Adelaide.

\section{References}

Barone J, DeLara J, Cummings KB and Ward WS (1994) DNA organisation in human spermatozoa Journal of Andrology 15 139-144

Bedford JM and Calvin HI (1974) The occurrence and possible functional significance of -S-S-crosslinks in sperm heads, with particular reference to eutherian mammals Journal of Experimental Zoology 188 137-156

Bedford JM and Hoskins DD (1990) The mammalian spermatozoon: morphology, biochemistry and physiology. In Marshall's Physiology of Reproduction Vol 2: Reproduction in the Male (4th Edn) pp 379-568 Ed. GE Lamming. Churchill Livingstone, Edinburgh

Bedford JM, Bent MI and Calvin HI (1973a) Variations in structural character and stability of the nuclear chromatin in morphologically normal human spermatozoa Journal of Reproduction and Fertility $3319-29$

Bedford JM, Calvin H and Cooper GW (1973b) The maturation of spermatozoa in the human epididymis Journal of Reproduction and Fertility Supplement 18 199-213

Bishop MWH and Austin CR (1957) Mammalian spermatozoa Endeavour 16 $137-150$

Blazak WF and Overstreet JW (1982) Instability of nuclear chromatin in the ejaculated spermatozoa of fertile men Journal of Reproduction and Fertility 65 331-339

Breed WG (1983) Variation in sperm morphology in the Australian rodent genus Pseudomys. Cell and Tissue Research $229611-625$

Breed WG (1993) Novel organization of the spermatozoon in two species of murid rodents from southern Asia Journal of Reproduction and Fertility 99 149-158

Breed WG (1995) Spermatozoa of murid rodents from Africa: morphological diversity and evolutionary trends Journal of Zoology London 237 625-651

Breed WG Evolution of sperm head morphology in Australasian murid rodents Australian Journal of Zoology (in press)

Breed WG, Cox GA, Leigh CM and Hawkins P (1988) Sperm head structure of a murid rodent from Southern Africa: the red veld rat Aethomys chrysophilus. Gamete Research 19 191-202

Burruel VR, Yanagimachi R and Whitten WK (1996) Normal mice develop from oocytes injected with spermatozoa with grossly misshapen heads Biology of Reproduction 55 709-714

Calvin HI (1976) Comparative analysis of the nuclear basic proteins in rat, human, guinea pig, mouse and rabbit spermatozoa Biochemica et Biophysica Acta 434 377-389

Calvin HI and Bedford JM (1971) Formation of disulphide bonds in the nucleus and accessory structures of mammalian spermatozoa during maturation in the epididymis Journal of Reproduction and Fertility Supplement 13 65-75

Chimimba CT (1997) A systematic revision of southern African Aethomys Thomas, 1915 (Rodentia: Muridae) PhD Thesis, University of Pretoria, Pretoria, South Africa

Clouthier DE, Avarbock MR, Maika SD, Hammer RE and Brinster RL (1996) Rat spermatogenesis in mouse testes Nature 381 418-421

DeGraaff G (1981) The Rodents of Southern Africa Butterworths, Durban
Eddy EM and O'Brien DA (1994) The spermatozoon. In The Physiology of Reproduction (2nd Edn) pp 29-77 Eds E Knobil and JD Neill. Raven Press, New York

Fawcett DW (1970) A comparative view of sperm ultrastructure Biology of Reproduction Supplement 2 90-127

Finch JT and Klug A (1976) A solenoid model for superstructure in chromatin Proceedings of the National Academy of Sciences USA 73 1897-1901

Friend GF (1936) The sperms of British Muridae Quarterly Journal of Microscopical Science 78 415-443

Gordon DH and Watson CRB (1986) Identification of cryptic species of rodents (Mastomys, Aethomys, Saccostomus) in the Kruger National Park South African Journal of Zoology 21 95-97

Koehler JK, Würschmidt U and Larsen MP (1983) Nuclear and chromatin structure in rat spermatozoa Gameie Research 8 357-370

Mahi CA and Yanagimachi R (1975) Induction of nuclear decondensation of mammalian spermatozoa in vitro. Journal of Reproduction and Fertility $\mathbf{4 4}$ 293-296

Perreault SD, Barbee RR, Elstein DH, Zucker RM and Keefer CL (1988) Interspecific differences in the stability of mammalian sperm nuclei assessed in vivo by sperm microinjection and in vitro by flow cytometry Biology of Reproduction 39 157-167

Rhim JA, Connor W, Dixon GH, Harendza CJ, Evenson DP, Palmiter RD and Brinster RL (1995) Expression of an avian protamine in transgenic mice disrupts chromatin structure in spermatozoa Biology of Reproduction 52 $20-32$

Seuanez HN, Carothers AD, Martin DE and Short RV (1977) Morphological abnormalities in spermatozoa of man and great apes Nature 270 345-347

Skinner JD and Smithers RHN (1990) The Mammals of the Southern African Region (2nd Edn) pp 1-769 University of Pretoria Press, Pretoria

Visser DS and Robinson TJ (1987) Systematic implications of spermatozoon and bacula morphology on the South African Aethomys. Mammalia 51 447-454

Ward WS (1993) Deoxyribonucleic acid loop domain tertiary structure in mammalian spermatozoa Biology of Reproduction 48 1193-1201

Ward WS and Coffey DS (1989) Identification of a sperm nuclear annulus in sperm Biology of Reproduction 41 361-370

Ward WS and Coffey DS (1991) DNA packaging and organisation in mammalian spermatozoa: comparison with somatic cells Biology of Reproduction 44 569-574

Wildt DE (1994) Endangered species spermatozoa; diversity, research and conservation. In Function of Somatic Cells in the Testis pp 1-24 Ed. A Bartke. Springer-Verlag, New York

Wildt DE, Bush M, Howard JG, O'Brien SJ, Meltzer D, van Dyk A, Ebedes $\mathrm{H}$ and Brand DJ (1983) Unique seminal quality in the South African cheetah and a comparative evaluation in the domestic cat Biology of Reproduction $\mathbf{2 9}$ $1019-1028$

Wildt DE, Phillips LG, Simmons LG, Chakraborky PK, Brown JL, Howard JG, Teare A and Bush M (1988) A comparative evaluation of ejaculate and hormonal characteristics of the captive male cheetah, tiger, leopard and puma Biology of Reproduction 38 245-255

Yanagimachi R (1994) Mammalian fertilization. In The Physiology of Reproduction (2nd Edn) pp 189-317 Eds E Knobil and JD Neill. Raven Press, New York

Zirkin BR, Soucek DA, Chang TSK and Perreault SD (1985) In vitro and in vivo studies of mammalian sperm nuclear decondensation Gamete Research $\mathbf{1 1}$ 349-365 